$$
\begin{aligned}
& \text { IN } 30 \\
& 31721
\end{aligned}
$$

THE CRACK PROBLEM IN BONDED NONHOMOGENEOUS MATERIALS

by

F. Erdogan

A.C. Kaya

P.F. Joseph

Lehigh University

Bethlehem, PA 18015

November 1988

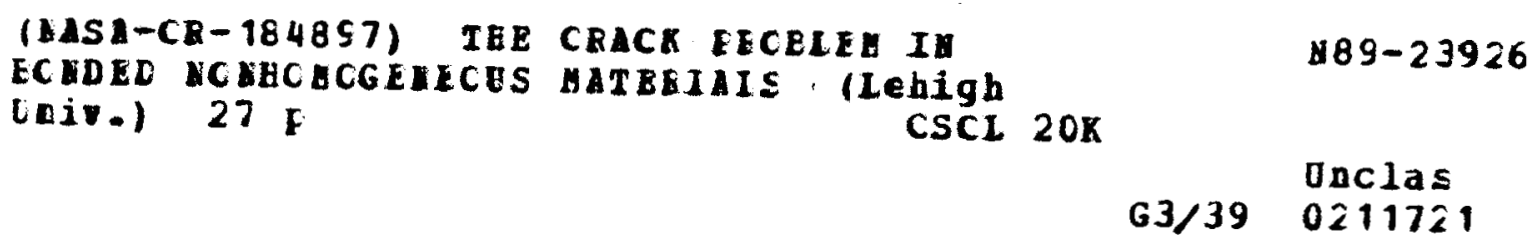

THE NATIONAL AERONAUTICS AND SPACE ADMINISTRATION

GRANT NAG-1-713 


\title{
THE CRACK PROBLEM IN BONDED NONHOMOGENEOUS MATERIALS
}

by

\author{
F. Erdogan, A.C. Kaya ${ }^{1}$ and P.F. Joseph \\ Lehigh University, Bethlehem, PA 18015
}

\begin{abstract}
In this paper the plane elasticity problem for two bonded half planes containing a crack perpendicular to the interface is considered. The primary objective of the paper is to study the effect of very steep variations in the material properties near the diffusion plane on the singular behavior of the stresses and stress intensity factors. The two materials are, thus, assumed to have the shear moduli $\mu_{0}$ and $\mu_{0} \exp (\beta \mathrm{x}), \mathrm{x}=0$ being the diffusion plane. Of particular interest is the examination of the nature of stress singularity near a crack tip terminating at the interface where the shear modulus has a discontinuous derivative. The results show that, unlike the crack problem in piecewise homogeneous materials for which the singularity is of the form $\mathrm{r}^{-\alpha}, 0<\alpha<1$, in this problem the stresses have a standard square-root singularity regardless of the location of the crack tip. The nonhomogeneity constant $\beta$ has, however, considerable influence on the stress intensity factors.
\end{abstract}

\section{Introduction}

From the viewpoint of mechanical failure in diffusion bonded materials and other adhesively bonded structures one of the most important problems is, along with the fracture characterization of the interfacial zones, a proper mechanical modelling of these zones and the development of suitable techniques for solving the related crack problems. The significance of the problem lies in the fact that (i) regardless of the mechanism of binding at the atomic level, in many cases there is always a thermodynamically stable and readily distinguishable region between the two homogeneous materials, (ii) the interfacial regions are generally locations of higher concentration of stresses and microflaws, and (iii) in most material pairs the fracture toughness and the subcritical crack growth resistance of the interfacial zone tend to be lower than that of adjacent homogeneous materials. The standard continuum solid mechanics model for bonded materials has been the usual piecewise homogeneous medium with an ideal interface across which the physical properties of the medium exhibit a jump discontinuity. For many applications this model is quite adequate. However, there are two groups of fracture related

${ }^{1}$ Assistant Professor, Department of Mechanical Engineering, Middle East Technical University, Ankara, Turkey 
problems in which the model leads to some physically anomalous results and may require modifications. The first is the complex power singularty at the tip of an interface crack giving oscilations in stresses and crack opening displacements very near the crack tip (e.g., Muskhelishvili (1953a), Erdogan (1965), England (1965), Rice and Sih (1965)). A crack tip contact model has been proposed to eliminate these oscillations within the context of the linear elastic theory, Comninou (1977), Atkinson (1977).

The second problem deals with a crack intersecting the interface. In-this case it is known that the stress state has a power singularity of the form $r^{-\alpha}$ with $\alpha \neq 1 / 2$ and $0<\alpha<1$, Cook and Erdogan (1972), Erdogan and Cook (1974), Erdogan and Biricikoglu (1973). The physically objectionable feature of this result is that because of the nonsquare-root singularity the propagating crack would not remain self-similar, and consequently the standard energy balance based fracture mechanics techniques would not be applicable to problems in bonded materials that involve cracks intersecting the interfaces.

The ideal interface model may be improved by considering the interdiffusion nature of the bonding process. In some cases the resulting reaction zone may be very thin and in others it may consist of intermediate phases or intermetallics with mechanical properties substantially different than the adjacent materials. In either case the microprobe line scans seem to indicate that in the interfacial region the percent distribution of atoms from one phase to the next is very steep but, nevertheless, continuous. Thus, in diffusion bonded materials one could replace the ideal interface requiring a jump discontinuity in physical properties across the boundary by a thin nonhomogeneous layer with highly steep variations in the mechanical properties. One may further assume that the elastic parameters of the composite medium are continuous functions of the space coordinates throughout the medium having discontinuous derivatives along the boundaries of the interfacial region. The analytical questions raised by this material model concern the effect of the material nonhomogeneity, particulary of the "kinks" in the distribution of the elastic parameters on the nature of the crack tip singularities. The crack problem for an infinite nonhomogeneous medium was considered by Delale and Erdogan (1983) who showed that as expected the standard square-root singularity at the crack tips is maintained if the material parameters are continuous and differentiable functions.

Regarding the physical anomalies that follow from the piecewise homogeneous material model, the first question deals with the nature of singularities for cracks lying along the kink lines of the elastic property distribution. This question was recently considered by Delale and Erdogan (1988a) who showed that for these "interface" cracks not only the square-root behavior of singularity but also the angular distribution of stresses very near the crack tips turn out to be identical to that found for the homogeneous materials. Further results for a crack in a nonhomogeneous interfacial zone are given by Delale and Erdogan (1988b). The second basic question in introducing the nonhomogeneous interfacial zone model is concerned 
with a crack intersecting the interface, specifically with the nature of stress singularity for a crack terminating at the kink line of the elastic property distribution. The problem under anti-plane shear loading was considered by Erdogan (1985) who showed that the square-root nature of stress singularity remains unaffected by the material nonhomogeneity provided the shear modulus is a continuous but not necessarily a differentiable function near and at the crack tip. The behavior of stress singularity in the corresponding plane strain problem is one of the remaining unresolved questions that will be considered in this paper.

\section{Formulation of the Crack Problem}

Consider the plane elasticity problem shown in Fig. 1 where a homogeneous half plane containing a crack perpendicular to the boundary is bonded to a nonhomogeneous half plane. For the purpose of examining the nature of crack tip singularity and for studying the effect of material nonhomogeneity on the stress intensity factors, it will be assumed that the elastic properties of the two materials are given by

$$
\mu_{1}(\mathrm{x})=\mu_{\mathrm{o}} \mathrm{e}^{\beta \mathrm{x}}, \mu_{2}=\mu_{\circ}, \kappa_{1}=\kappa_{2}=\kappa
$$

where $\mu$ is the shear modulus, $\kappa=3-4 \nu$ for plane strain and $\kappa=(3-\nu) /(1+\nu)$ for generalized plane stress, $\nu$ being the Poisson's ratio. With (1) the Navier's equations may be expressed as

$$
\begin{aligned}
& \nabla^{2} u_{i}+\frac{2}{\kappa-1}\left(\frac{\partial^{2} u_{i}}{\partial x^{2}}+\frac{\partial^{2} v_{i}}{\partial x \partial y}\right)+2 \beta \frac{\partial u_{i}}{\partial x}+\beta\left(\frac{3-\kappa}{\kappa-1}\right)\left(\frac{\partial u_{i}}{\partial x}+\frac{\partial v_{i}}{\partial y}\right)=0 \\
& \nabla^{2} v_{i}+\frac{2}{\kappa-1}\left(\frac{\partial^{2} v_{i}}{\partial y^{2}}+\frac{\partial^{2} u_{i}}{\partial x \partial y}\right)+\beta\left(\frac{\partial u_{i}}{\partial y}+\frac{\partial v_{i}}{\partial x}\right)=0,(i=1,2)
\end{aligned}
$$

where $u_{i}$ and $v_{i},(i=1,2)$ are the $x$ and $y$ components of the displacement and $\beta \neq 0$ for $i=1$ or $x<0$ and $\beta=0$ for $i=2$ or $x>0$ (Fig. 1). Referring to Fig. 1 , it will be assumed that $y=0$ plane is a plane of symmetry for the external loads as well as the geometry. It will also be assumed that the problem in the absence of the crack under the actual loading conditions has been solved and $\sigma_{2 y y}^{h}(x, 0)$ has been determined. Thus, the important results concerning the crack problem may be obtained by considering only the perturbation problem for $y>0$ in which the normal traction $p(x)=-\sigma_{2 y y}^{h}(x, 0)$ is the only external load. Equation (2) must then be solved under the following conditions:

$$
\begin{aligned}
& u_{1}(0, y)=u_{2}(0, y), v_{1}(0, y)=v_{2}(0, y), 0<y<\infty, \\
& \sigma_{1 x x}(0, y)=\sigma_{2 x x}(0, y), \sigma_{1 x y}(0, y)=\sigma_{2 x y}(0, y), 0<y<\infty,
\end{aligned}
$$




$$
\begin{aligned}
& \mathrm{v}_{1}(\mathrm{x}, 0)=0, \sigma_{1 \mathrm{xy}}(\mathrm{x}, 0)=0,-\infty<\mathrm{x}<0, \\
& \sigma_{2 x y}(\mathrm{x}, 0)=0,0<\mathrm{x}<\infty, \\
& \mathrm{v}_{2}(\mathrm{x}, 0)=0,0<\mathrm{x}<\mathrm{a}, \mathrm{b}<\mathrm{x}<\infty, \\
& \sigma_{2 y y}(\mathrm{x},+0)=\mathrm{p}(\mathrm{x}), \mathrm{a}<\mathrm{x}<\mathrm{b} .
\end{aligned}
$$

The solution of (2) may now be written as

$$
\begin{aligned}
& \mathrm{u}_{1}(\mathrm{x}, \mathrm{y})=\frac{2}{\pi} \int_{0}^{\infty} \mathrm{f}(\mathrm{x}, \alpha) \cos \alpha \mathrm{yd} \alpha, \\
& \left.\mathrm{v}_{1}(\mathrm{x}, \mathrm{y})=\frac{2}{\pi} \int_{0}^{\infty} \mathrm{g}(\mathrm{x}, \alpha) \sin \alpha \mathrm{yd} \alpha,-\infty<\mathrm{x}, \mathrm{b}\right) \\
& \mathrm{u}_{2}(\mathrm{x}, \mathrm{y})=\frac{2}{\pi} \int_{0}^{\infty} \mathrm{f}_{1}(\mathrm{x}, \alpha) \cos \alpha \mathrm{yd} \alpha+\frac{1}{2 \pi} \int_{-\infty}^{\infty} \mathrm{f}_{2}(\mathrm{y}, \alpha) \mathrm{e}^{-\mathrm{i} \alpha \mathrm{x}} \mathrm{d} \alpha, \\
& \mathrm{v}_{2}(\mathrm{x}, \mathrm{y})=\frac{2}{\pi} \int_{0}^{\infty} \mathrm{g}_{1}(\mathrm{x}, \alpha) \sin \alpha \mathrm{yd} \alpha+\frac{1}{2 \pi} \int_{-\infty}^{\infty} \mathrm{g}_{2}(\mathrm{y}, \alpha) \mathrm{e}^{-\mathrm{i} \alpha \mathrm{x}} \mathrm{d} \alpha, \quad 0<\mathrm{x}<\infty, 0<\mathrm{y}<\infty .(9 \mathrm{a}, \mathrm{b})
\end{aligned}
$$

By substituting from (8) into (2) it can be shown that

$$
f(x, \alpha)=\sum_{j=1}^{4} m_{j} D_{j}(\alpha) e^{n_{j} x}, g(x, \alpha)=\sum_{j=1}^{4} D_{j}(\alpha) e^{n_{j} x},
$$

where $D_{1}, \ldots, D_{4}$ are unknown, $n_{1}, \ldots, n_{4}$ are the roots of the characteristic equation

$$
\mathrm{n}^{4}+2 \beta \mathrm{n}^{3}+\left(\beta^{2}-2 \alpha^{2}\right) \mathrm{n}^{2}-2 \beta \alpha^{2} \mathrm{n}+\left(\frac{3-\kappa}{1+\kappa}\right) \beta^{2} \alpha^{2}+\alpha^{4}=0
$$

and $m_{1}, \ldots, m_{4}$ are given by

$$
m_{j}=\frac{n_{j}^{2}+\beta n_{j}\left(1+a_{0}\right) \alpha^{2}}{\left(a_{0} n_{j}+\beta\right) \alpha}, a_{0}=\frac{2}{\kappa-1}, j=1, \ldots, 4 \text {. }
$$

By rewriting (11) in the following form

$$
\left(n^{2}+\beta n-\alpha^{2}\right)^{2}+\left(\frac{3-\kappa}{1+\kappa}\right) \beta^{2} \alpha^{2}=0,
$$

the roots may be obtained as

$$
\mathrm{n}_{1}=\frac{1}{2}(-\beta+\mathrm{R} \cos \gamma)+\frac{\mathrm{i}}{2} \mathrm{R} \sin \gamma, \mathrm{n}_{2}=\overline{\mathrm{n}}_{1},
$$




$$
\begin{aligned}
& \mathrm{n}_{3}=\frac{1}{2}(-\beta-\mathrm{R} \cos \gamma)+\frac{\mathrm{i}}{2} \mathrm{Rsin} \gamma, \mathrm{n}_{4}=\overline{\mathrm{n}}_{3}, \\
& \mathrm{R}=\left[\left(\beta^{2}+4 \alpha^{2}\right)^{2}+16 \beta^{2} \alpha^{2}\left(\frac{3-\kappa}{1+\kappa}\right)\right]^{1 / 4}, \tan 2 \gamma=\frac{4 \beta \alpha \sqrt{\frac{3-\kappa}{1+\kappa}}}{\beta^{2}+4 \alpha^{2}}
\end{aligned}
$$

where we note that $R$ is always positive and

$$
\operatorname{Re}\left(\mathrm{n}_{1}, \mathrm{n}_{2}\right)>0, \operatorname{Re}\left(\mathrm{n}_{3}, \mathrm{n}_{4}\right)<0
$$

Observing that for the material $1 x<0$, by using the regularity conditions at infinity we obtain

$$
\begin{aligned}
& \mathrm{u}_{1}(\mathrm{x}, \mathrm{y})=\frac{2}{\pi} \int_{0}^{\infty} \sum_{1}^{2} \mathrm{~m}_{\mathrm{j}}(\alpha) \mathrm{D}_{\mathrm{j}}(\alpha) \mathrm{e}^{\mathrm{n}_{\mathrm{j}}(\alpha) \mathrm{x}} \cos \alpha \mathrm{yd} \alpha, \\
& \mathrm{v}_{1}(\mathrm{x}, \mathrm{y})=\frac{2}{\pi} \int_{0}^{\infty} \sum_{1}^{2} \mathrm{D}_{\mathrm{j}}(\alpha) \mathrm{e}^{\mathrm{n}_{\mathrm{j}}(\alpha) \mathrm{x}} \sin \alpha \mathrm{yd} \alpha,-\infty<\mathrm{x}<0,0<\mathrm{y}<\infty, \\
& \sigma_{1 \mathrm{xx}}(\mathrm{x}, \mathrm{y})=\frac{\mu_{\mathrm{o}} \mathrm{e}^{\beta \mathrm{x}}}{\kappa-1} \frac{2}{\pi} \int_{0}^{\infty} \sum_{1}^{2}\left[(1+\kappa) \mathrm{m}_{\mathrm{j}} \mathrm{n}_{\mathrm{j}}+(3-\kappa) \alpha\right] \mathrm{D}_{\mathrm{j}} \mathrm{e}^{\mathrm{n}_{\mathrm{j}} \mathrm{x}} \cos \alpha \mathrm{yd} \alpha, \\
& \sigma_{1 \mathrm{yy}}(\mathrm{x}, \mathrm{y})=\frac{\mu_{\mathrm{o}} \mathrm{e}^{\beta \mathrm{x}}}{\kappa-1} \frac{2}{\pi} \int_{0}^{\infty} \sum_{1}^{2}\left[(1+\kappa) \alpha+(3-\kappa) \mathrm{m}_{\mathrm{j}} \mathrm{n}_{\mathrm{j}}\right] \mathrm{D}_{\mathrm{j}} \mathrm{e}^{\mathrm{n}_{\mathrm{j}} \mathrm{x}} \cos \alpha \mathrm{yd} \alpha, \\
& \sigma_{1 \mathrm{xy}}(\mathrm{x}, \mathrm{y})=\mu_{\mathrm{o}} \mathrm{e}^{\beta \mathrm{x}} \frac{2}{\pi} \int_{0}^{\infty} \sum_{1}^{2}\left(\mathrm{n}_{\mathrm{j}}-\alpha \mathrm{m}_{\mathrm{j}}\right) \mathrm{D}_{\mathrm{j}} \mathrm{e}^{\mathrm{n}_{\mathrm{j}} \mathrm{x}} \sin \alpha \mathrm{yd} \alpha,-\infty<\mathrm{x}<0,0<\mathrm{y}<\infty,
\end{aligned}
$$

Similarly, substituting from (9) into (2) and letting $\beta=0$, we find

$$
\begin{aligned}
\mathrm{u}_{2}(\mathrm{z}, \mathrm{y}) & =\frac{2}{\pi} \int_{0}^{\infty}\left[\mathrm{A}_{1}+\left(\mathrm{x}+\frac{\kappa}{\alpha}\right) \mathrm{A}_{2}\right] \mathrm{e}^{-\alpha \mathrm{x}} \cos \alpha \mathrm{yd} \alpha \\
& +\frac{1}{2 \pi} \int_{-\infty}^{\infty}\left(\frac{1-\kappa}{2|\alpha|}+\mathrm{y}\right) \mathrm{A}_{3} \mathrm{e}^{-|\alpha| \mathrm{y}-\mathrm{i} \alpha \mathrm{x}} \mathrm{d} \alpha \\
\mathrm{v}_{2}(\mathrm{x}, \mathrm{y}) & =\frac{2}{\pi} \int_{0}^{\infty}\left(\mathrm{A}_{1}+\mathrm{A}_{2} \mathrm{x}\right) \mathrm{e}^{-\alpha \mathrm{x}} \sin \alpha \mathrm{y} \\
& -\frac{\mathrm{i}}{2 \pi} \int_{-\infty}^{\infty}\left(\frac{1+\kappa}{2|\alpha|}+\mathrm{y}\right) \frac{|\alpha|}{\alpha} \mathrm{A}_{3} \mathrm{e}^{-|\alpha| \mathrm{y}-\mathrm{i} \alpha \mathrm{x}} \mathrm{d} \alpha, 0<\mathrm{x}<\infty, 0<\mathrm{y}<\infty
\end{aligned}
$$




$$
\begin{gathered}
\frac{1}{2 \mu_{\mathrm{o}}} \sigma_{2 \mathrm{xx}}(\mathrm{x}, \mathrm{y})=-\frac{2}{\pi} \int_{0}^{\infty}\left[\alpha \mathrm{A}_{1}+\left(\alpha \mathrm{x}+\frac{\kappa+1}{2}\right) \mathrm{A}_{2}\right] \mathrm{e}^{-\alpha \mathrm{x}} \cos \alpha \mathrm{yd} \alpha \\
+\frac{\mathrm{i}}{2 \pi} \int_{-\infty}^{\infty}\left(\frac{|\alpha|}{\alpha}-\alpha \mathrm{y}\right) \mathrm{A}_{3} \mathrm{e}^{-|\alpha| \mathrm{y}-\mathrm{i} \alpha \mathrm{x}} \mathrm{d} \alpha, \\
\frac{1}{2 \mu_{\mathrm{o}}} \sigma_{2 \mathrm{yy}}(\mathrm{x}, \mathrm{y})=\frac{2}{\pi} \int_{0}^{\infty}\left[\alpha \mathrm{A}_{1}+\left(\alpha \mathrm{x}+\frac{\kappa-3}{2}\right) \mathrm{A}_{2}\right] \mathrm{e}^{-\alpha \mathrm{x}} \cos \alpha \mathrm{yd} \alpha \\
+\frac{\mathrm{i}}{2 \pi} \int_{-\infty}^{\infty}\left(\frac{|\alpha|}{\alpha}+\alpha \mathrm{y}\right) \mathrm{A}_{3} \mathrm{e}^{-|\alpha| \mathrm{y}-\mathrm{i} \alpha \mathrm{x}} \mathrm{d} \alpha, \\
\frac{1}{2 \mu_{\mathrm{o}}} \sigma_{2 \mathrm{xy}}(\mathrm{x}, \mathrm{y})=-\frac{2}{\pi} \int_{0}^{\infty}\left[\alpha \mathrm{A}_{1}+\left(\alpha \mathrm{x}+\frac{\kappa-1}{2}\right) \mathrm{A}_{2}\right] \mathrm{e}^{-\alpha \mathrm{x} \sin \alpha \mathrm{yd} \alpha} \\
-\frac{2}{\pi} \int_{0}^{\infty}|\alpha| \mathrm{y} \mathrm{A}_{3} \mathrm{e}^{-|\alpha| \mathrm{y}-\mathrm{i} \alpha \mathrm{x}} \mathrm{d} \alpha, 0<\mathrm{x}<\infty, 0<\mathrm{y}<\infty
\end{gathered}
$$

where $A_{1}, A_{2}, A_{3}$ are unknown functions.

It may be seen that because of the assumed symmetry in (8) and (9), the conditions (5) and (6) are identically satisfied. The remaining five conditions (3), (4) and (7) may then be used to determine the unknowns $D_{1}, D_{2}, A_{1}, A_{2}$, and $A_{3}$.

\section{Derivation of the Integral Equation}

To reduce the problem to an integral equation we first introduce

$$
\phi(x)=\frac{\partial}{\partial x} v_{2}(x, 0), a<x<b
$$

From (22) and (20b) it then follows that

$$
A_{3}(\alpha)=-\frac{2}{\kappa-1} \int_{a}^{b} \phi(t) e^{i \alpha t} d t
$$

The four homogeneous conditions (3) and (4) may now be used to express $D_{1}, D_{2}, A_{1}$ and $A_{2}$ in terms of $\phi$, which may then be determined by using (7b). The expressions of $D_{1}, D_{2}, A_{1}$ and $\mathrm{A}_{2}$ are given in Appendix $\mathrm{A}$.

In considering the boundary condition (7a), from (21b) and (23) the contribution of the second term on the right hand side of (21b) may easily be evaluated as follows:

$$
\begin{aligned}
\lim _{\mathrm{y} \rightarrow+0} \frac{2 \mu_{\mathrm{o}} \mathrm{i}}{2 \pi} \int_{-\infty}^{\infty}\left(\frac{|\alpha|}{\alpha}+\alpha \mathrm{y}\right) \mathrm{A}_{3}(\alpha) \mathrm{e}^{-|\alpha| \mathrm{y}-\mathrm{i} \alpha \mathrm{x}} \mathrm{d} \alpha \\
\quad=\frac{4 \mu_{\mathrm{o}}}{\pi(\kappa+1)} \lim _{\mathrm{y} \rightarrow+0} \int_{\mathrm{a}}^{\mathrm{b}}\left[\frac{\mathrm{t}-\mathrm{x}}{(\mathrm{t}-\mathrm{x})^{2}+\mathrm{y}^{2}}+\frac{2(\mathrm{t}-\mathrm{x}) \mathrm{y}^{2}}{\left[(\mathrm{t}-\mathrm{x})^{2}+\mathrm{y}^{2}\right]^{2}}\right] \phi(\mathrm{t}) \mathrm{dt}
\end{aligned}
$$




$$
=\frac{4 \mu_{\mathrm{o}}}{\pi(\kappa+1)} \int_{\mathrm{a}}^{\mathrm{b}} \frac{\phi(\mathrm{t}) \mathrm{dt}}{\mathrm{t}-\mathrm{x}} .
$$

Evaluating the contribution coming from the first term on the right hand side of (21b) by making use of the results given in Appendix A, the condition (7b) may now be expressed as

$$
\frac{1}{\pi} \int_{a}^{b}\left[\frac{1}{t-x}+K(x, t)\right] \phi(t) d t=\frac{\kappa+1}{4 \mu_{0}} p(x), a<x<b
$$

where the kernel $\mathrm{K}$ is given by

$$
\begin{aligned}
& \mathrm{K}(\mathrm{x}, \mathrm{t})=\int_{0}^{\infty} \mathrm{h}(\alpha, \mathrm{x}, \mathrm{t}) \mathrm{e}^{-\alpha(\mathrm{t}+\mathrm{x})} \mathrm{d} \alpha \\
& \mathrm{h}(\alpha, \mathrm{x}, \mathrm{t})=\mathrm{h}_{1}(\alpha)+\mathrm{xh}_{2}(\alpha)+\mathrm{th}_{3}(\alpha)+\operatorname{txh}_{4}(\alpha), \\
& \mathrm{h}_{1}(\alpha)=(\kappa+1)\left[\alpha \mathrm{p}_{3}(\alpha)+\frac{\kappa-3}{2} \mathrm{p}_{4}(\alpha)\right] \\
& \mathrm{h}_{2}(\alpha)=(\kappa+1) \alpha \mathrm{p}_{4}(\alpha) \\
& \mathrm{h}_{3}(\alpha)=(\kappa+1)\left[\alpha \mathrm{q}_{3}(\alpha)+\frac{\kappa-3}{2} \mathrm{q}_{4}(\alpha)\right] \\
& \mathrm{h}_{4}(\alpha)=(\kappa+1) \alpha \mathrm{q}_{4}(\alpha) .
\end{aligned}
$$

The functions $p_{3}, p_{4}, q_{3}$ and $q_{4}$ may also be found in Appendix $A$.

Before proceeding with the examination of the asymptotic behavior of the kernel $K(x, t)$, two special cases may be considered. The first is the case of $\beta=0$ for which the problem is one of a homogeneous infinite plane containing a single crack. For $\beta \rightarrow 0$ it may easily be shown that $\mathrm{A} \rightarrow \alpha, \mathrm{B} \rightarrow 0, \mathrm{C} \rightarrow-1, \mathrm{D} \rightarrow 0$ and, consequently $\mathrm{K}(\mathrm{x}, \mathrm{t})$ vanishes giving (see Appendix $\mathrm{A}$ )

$$
\frac{1}{\pi} \int_{a}^{b} \frac{\phi(t)}{t-x} d t=\frac{1+\kappa}{4 \mu_{0}} p(x), a<x<b
$$

which is the expected result. In the second special case we let $\beta=\infty$ which, from Fig. 1 and $\mu_{1}(x)=\mu_{0} \exp (\beta x),(x<0)$, is seen to correspond to $\mu_{1}=0$. The problem would then be one of a homogeneous half plane having a crack perpendicular to the boundary. From (12), (14), (16), (A15) and (A16) it can be shown that for $\beta \rightarrow \infty$

$$
\mathrm{A} \rightarrow 0, \mathrm{~B} \rightarrow \alpha \sqrt{\frac{3-\kappa}{1+\kappa}}, \mathrm{C} \rightarrow 0, \mathrm{D} \rightarrow \sqrt{\frac{3-\kappa}{1+\kappa}}
$$

Thus, by substituting from Appendix A and (30) into (28) and (27) we obtain 


$$
h(\alpha, t, x) \rightarrow\left(2-\alpha x-3 \alpha t+2 \alpha^{2} t x\right)
$$

From (26) and (31) it then follows that

$$
K(x, t)=-\frac{1}{t+x}+\frac{6 x}{(t+x)^{2}}-\frac{4 x^{2}}{(t+x)^{3}}
$$

which is the expected half plane result (Cook and Erdogan, 1972).

In piecewise homogeneous materials having the same crack geometry the kernel $\mathrm{K}$ has a form quite similar to (32), namely (Erdogan and Biricikoglu, 1973)

$$
K(x, t)=\frac{b_{1}}{t+x}+\frac{b_{2} x}{(t+x)^{2}}+\frac{b_{3} x^{2}}{(t+x)^{3}}
$$

where $b_{1}, b_{2}$, and $b_{3}$ are material property dependent constants. Note that if the crack tip touches the interface, i.e., for $\mathrm{a}=0, \mathrm{~K}$ would become unbounded as $\mathrm{x}$ and $\mathrm{t}$ approach zero and hence would contribute to the singular behavior of the solution. It is then clear that in order to examine the singular nature of the solution of (25), the asymptotic behavior of $\mathrm{K}$ must be investigated. Since the function $h$ in (26) is integrable at $\alpha=0$ and bounded elsewhere, any possible divergence in $K$ must be due to the asymptotic behavior of $h$ as $\alpha \rightarrow \infty$. Referring now to Appendix B, for $\alpha \rightarrow \infty$ we obtain

$$
\mathrm{h}(\alpha, \mathrm{x}, \mathrm{t}) \rightarrow \mathrm{h}_{\infty}(\alpha, \mathrm{x}, \mathrm{t})=\frac{\mathrm{d}_{1}}{\alpha}+\left(\mathrm{d}_{2}+\frac{\mathrm{d}_{3}}{\alpha}\right) \mathrm{t}+\left(\mathrm{d}_{4} \alpha+\mathrm{d}_{5}+\frac{\mathrm{d}_{6}}{\alpha}\right) \mathrm{tx}
$$

where

$$
d_{1}=\frac{\beta}{4}, d_{2}=-d_{4}=-\frac{\beta}{1+\kappa}, d_{3}=-d_{5}=\frac{(1-\kappa) \beta^{2}}{2(1+\kappa)^{2}}, d_{6}=-\frac{\kappa(3-\kappa) \beta^{3}}{4(1+\kappa)^{3}}
$$

By subtracting and adding $h_{\infty}$ from and to $h$ in (26) and by evaluating the integrals, it may easily be shown that

$$
\begin{aligned}
k(x, t) & =k_{1}(x, t)+k_{2}(x, t)+k_{3}(x, t) \\
k_{1}(x, t) & =\int_{0}^{\infty}\left(d_{2} t+d_{5} t x+d_{4} \alpha t x\right) e^{-\alpha(t+x)} d \alpha \\
& =d_{2} \frac{t}{t+x}+d_{5} \frac{t x}{t+x}+d_{4} \frac{t x}{(t+x)^{2}},
\end{aligned}
$$




$$
\begin{aligned}
k_{2}(x, t) & =-\left(d_{1}+d_{3} t+d_{6} t x\right) E i\left[-\alpha_{0}(t+x)\right] \\
k_{3}(x, t) & =\int_{0}^{\alpha_{0}}\left[h(\alpha, x, t)-\left(d_{2} t+d_{5} t x+d_{4} t x \alpha\right)\right] e^{-\alpha(t+x)} d \alpha \\
& +\int_{\alpha_{0}}^{\infty}\left[h(\alpha, x, t)-h_{\infty}(\alpha, x, t)\right] e^{-\alpha(t+x)} d \alpha
\end{aligned}
$$

where $\alpha_{0}$ is any positive constant ${ }^{2}$ and the exponential integral is defined by

$$
\begin{aligned}
& \operatorname{Ei}\left[-\alpha_{\mathrm{o}}(\mathrm{t}+\mathrm{x})\right]=-\int_{\alpha_{\mathrm{o}}}^{\infty} \frac{1}{\alpha} \mathrm{e}^{-\alpha(\mathrm{t}+\mathrm{x})} \mathrm{d} \alpha \\
& =\mathrm{C}_{\mathrm{o}}+\log \alpha_{\mathrm{o}}+\log (\mathrm{t}+\mathrm{x})+\sum_{\mathrm{k}=1}^{\infty} \frac{(-1)^{\mathrm{k}}(\mathrm{t}+\mathrm{x})^{\mathrm{k}} \alpha_{\mathrm{o}}{ }^{\mathrm{k}}}{\mathrm{k}(\mathrm{k} !)}, \\
& \alpha_{\circ}>0, \mathrm{t}+\mathrm{x}>0,
\end{aligned}
$$

and $\mathrm{C}_{\mathrm{O}}=0.5772156649$ is the Euler's constant.

By substituting from (36)-(38) into (25) we obtain

$$
\begin{aligned}
& \frac{1}{\pi} \int_{a}^{b}\left[\frac{1}{t-x}+\left(d_{4}-d_{2}\right) \frac{x}{t+x}-d_{5} \frac{x^{2}}{t+x}-d_{4} \frac{x^{2}}{(t+x)^{2}}\right] \phi(t) d t \\
& \quad+\frac{1}{\pi} \int_{a}^{b}\left[d_{2}+d_{5} x+k_{2}(x, t)+k_{3}(x, t)\right] \phi(t) d t=\frac{\kappa+1}{4 \mu_{0}} p(x),(a<x<b) .
\end{aligned}
$$

For $a>0(40)$ is an ordinary singular integral equation the dominant part of which has a simple Cauchy singularity. Its solution may, therefore, be expressed as (Muskhelishvili, 1953b)

$$
\phi(t)=\frac{F(t)}{\sqrt{(t-a)(b-t)}}, a<t<b
$$

where $\mathrm{F}$ is a bounded unknown function. For $\mathrm{a}=0$ the kernel of the second term in the lefthand side of (40) can still be treated as a Fredholm kernel and would not have any contribution to the singular behavior of $\phi(t)$. To examine the first term we let

$$
\phi(t)=\frac{F(t)}{t^{\omega}(b-t)^{\eta}}, 0<t<b, 0<\operatorname{Re}(\omega, \eta)<1,
$$

where $\mathrm{F}$ again is a bounded function. Defining the sectionally holomorphic function

${ }^{2}$ In numerical analysis rapid convergence is obtained by selecting $0<\alpha_{0}<1$. 


$$
\psi(\mathrm{z})=\frac{1}{\pi} \int_{0}^{\mathrm{b}} \frac{\phi(\mathrm{t})}{\mathrm{t}-\mathrm{z}} \mathrm{dt}
$$

it can be shown that (Muskhelishvili, 1953b)

$$
\psi(z)=\frac{F(0) e^{i \pi \omega}}{b^{\eta} \sin \pi \omega} \frac{1}{z^{\omega}}-\frac{F(b)}{b^{\omega} \sin \pi \eta} \frac{1}{(z-b)^{\eta}}+\psi_{o}(z)
$$

where $\psi_{o}(z)$ may have at most weaker singularities than $z^{-\omega}$ and $(z-b)^{-\eta}$ at $z=0$ and $z=b$, respectively. From (43) and (44) it can be shown that

$$
\frac{1}{\pi} \int_{0}^{b} \frac{\phi(t)}{t-x} d t=\frac{F(0) \cot \pi \omega}{b^{\eta}} \frac{1}{x^{\omega}}-\frac{F(b) \cot \pi \eta}{b^{\omega}} \frac{1}{(b-x)^{\eta}}+\psi_{1}(x), 0<x<b
$$

where at the end points the behavior of $\psi_{1}$ is the same as that of $\psi_{0}$. From (43) and (44) it can also be shown that near $x=0$ we have

$$
\frac{1}{\pi} \int_{0}^{b} \frac{\phi(t)}{t+x} d t=\psi(-x)=\frac{F(0)}{b^{\eta} \sin \pi \omega} \frac{1}{x^{\omega}}+\psi_{2}(x), 0<x<b
$$

where at $\mathrm{x}=0 \quad \psi_{2}$ again may have at most a weaker singularity than $\mathrm{x}^{\omega}$. From (46) it follows that

$$
\begin{aligned}
& \frac{1}{\pi} \int_{0}^{b} \frac{x^{k} \phi(t)}{t+x} d t=\frac{F(0)}{b^{\eta} \sin \pi \omega} x^{k-\omega}+x^{k} \psi_{2}(x), k=1,2, \ldots, 0<x<b \\
& \frac{1}{\pi} \int_{0}^{b} \frac{x^{2} \phi(t)}{(t+x)^{2}} d t=\frac{\omega F(0)}{b^{\eta} \sin \pi \omega} x^{1-\omega}-x^{2} \frac{d}{d x} \psi_{2}(x), 0<x<b .
\end{aligned}
$$

If we now substitute from (45), (47) and (48) into (40), first multiply both sides by $(b-x)^{\eta}$ and let $x=b$ then by $x^{\omega}$ and let $x=0$, and observe that $F(0) \neq 0, F(b) \neq 0$, we find

$$
\cot \pi \omega=0, \cot \pi \eta=0 .
$$

giving $\omega=\eta=1 / 2$. This is an important result indicating that the solution of the problem 
has a square-root singularity regardless of the location of the crack tip and the solution has the form as given by (41) for $a=0$ as well as $a>0^{3}$.

\section{The Solution and Results}

To solve the integral equation (25) we make a change in variable and define the following normalized quantities:

$$
\begin{aligned}
& t=\left(\frac{b-a}{2}\right) r+\frac{b+a}{2}, x=\left(\frac{b-a}{2}\right) s+\frac{b+a}{2}, \\
& \phi(t)=\frac{F(t)}{\sqrt{(t-a)(b-t)}}=\frac{G(r)}{\sqrt{1-r^{2}}}, a<t<b,-1<r<1, \\
& \left(\frac{b-a}{2}\right) K(x, t)=k(s, r), \\
& \frac{1+\kappa}{4 \mu_{o}} p(x)=Q(s) .
\end{aligned}
$$

Equation (25) then becomes

$$
\frac{1}{\pi} \int_{-1}^{1}\left[\frac{1}{r-s}+k(r, s)\right] \frac{G(r)}{\sqrt{1-r^{2}}} d r=Q(s),-1<s<1 .
$$

From (7a), (22) and (23) it is clear that the integral equation must be solved under the following single-valuedness condition:

$$
\int_{a}^{b} \phi(t) d t=0
$$

or

$$
\int_{-1}^{1} \frac{\mathrm{G}(\mathrm{r})}{\sqrt{1-\mathrm{r}^{2}}} \mathrm{dr}=0
$$

${ }^{3}$ Based on this and the previous results found by Delale and Erdogan (1983,1988a), one may now conjecture that in plane elasticity problems the material nonhomogeneity would have no influence on the nature of crack tip singularity regardless of the location and the orientation of the crack as long as the material parameters are continuous, but not necessarily differentiable, functions of the coordinates. This means that for a crack intersecting the interfacial region at an arbitrary angle, the complex power singularity predicted by the piecewise homogeneous material model for certain materials and crack orientations would also disappear and the square-root singularity at the crack tips would be the only singularity the solution may have. 
Equation (54) is solved by substituting

$$
G(r)=\sum_{0}^{\infty} a_{n} T_{n}(r)
$$

and by using a simple collocation scheme to reduce the resulting functional equation to an infinite system. First, from (56) and the orthogonality condition of Chebyshev polynomials $T_{n}$ it may be seen that

$$
a_{0}=0
$$

Thus, from (54), (57) and (58) by truncating the series and by using

$$
\begin{aligned}
& \frac{1}{\pi} \int_{-1}^{1} \frac{T n(r) d r}{(r-s) \sqrt{1-r^{2}}}=\left\{\begin{array}{c}
0, n=0 \\
U_{n-1}(s), n=1,2, \ldots,-1<s<1,
\end{array}\right. \\
& \sum_{1}^{N} a_{n}\left[U_{n-1}\left(s_{j}\right)+\frac{1}{\pi} \int_{-1}^{1} \frac{T_{n}(r) k\left(r, s_{j}\right)}{\sqrt{1-r^{2}}} d r\right]=Q\left(s_{j}\right), j=1, \ldots, N,
\end{aligned}
$$

where $U_{n}$ is the Chebyshev polynomial of the second kind. The collocation points $s_{j}$ may be selected as follows:

$$
T_{N}\left(s_{j}\right)=0, s_{j}=\cos \left[(2 j-1) \frac{\pi}{2 N}\right], j=1, \ldots, N
$$

Since (25) gives $\sigma_{2 y y}(x, 0)$ outside as well as inside the crack, substituting from (41) into (25) a simple asymptotic analysis would show that near the crack tips we have (see, for example, Erdogan, 1985)

$$
\begin{aligned}
& \sigma_{2 y z}(x, 0)=-\frac{4 \mu_{0}}{1+\kappa} \frac{F(b)}{\sqrt{(b-a)(x-b)}}+\sigma_{1}(x), \\
& \sigma_{2 y z}(x, 0)=\frac{4 \mu_{o}}{1+\kappa} \frac{F(a)}{\sqrt{(b-a)(a-x)}}+\sigma_{2}(x)
\end{aligned}
$$

where $\sigma_{1}$ and $\sigma_{2}$ represent the part of the solution that is bounded at $\mathrm{x}=\mathrm{b}$ and $\mathrm{x}=\mathrm{a}$, respectively. Thus, from (51) and (62) the mode I stress intensity factors defined by 


$$
k_{1}(a)=\lim _{x \rightarrow a} \sqrt{2(a-x)} \sigma_{1 y y}(x, 0), k_{1}(b)=\lim _{x \rightarrow b} \sqrt{2(x-b)} \sigma_{1 y y}(x, 0)
$$

may be expressed as ${ }^{4}$

$$
\mathbf{k}_{1}(\mathrm{a})=\frac{4 \mu_{\mathrm{o}}}{1+\kappa} \mathrm{G}(-1) \sqrt{\frac{\mathrm{b}-\mathrm{a}}{2}}, \mathrm{k}_{1}(\mathrm{~b})=-\frac{4 \mu_{\mathrm{o}}}{1+\kappa} \mathrm{G}(1) \sqrt{\frac{\mathrm{b}-\mathrm{a}}{2}}
$$

The main results obtained in this study regarding the stress intensity factors are shown in Table 1 , where it is assumed that

$$
\frac{\mathrm{b}-\mathrm{a}}{2}=\mathrm{c}, \frac{\mathrm{b}+\mathrm{a}}{2}=\mathrm{d}, \mathrm{p}(\mathrm{x})=-\sigma
$$

The uniform crack surface pressure $\sigma$ used in the example is realistic if the composite medium is subjected to a constant displacement loading in y direction away from the crack region. In the examples considered it is further assumed that $\nu=0.3$ and the plane strain conditions prevail. By examining the results given in the table a number of observations may be made. First from Fig. 1 and $\mu_{1}(x)=\mu_{0} \exp (\beta x)$ we note that for $\beta>0$ medium 2 is stiffer than medium 1 , hence the stress intensity factors would be expected and are seen to be greater than $\sigma \sqrt{c}$, the value for the corresponding homogeneous material having the same crack. For $\beta<0$ material 1 is the stiffer medium and consequently the stress intensity factors, as expected, turn out to be less than $\sigma \sqrt{c}$. These trends are also observed for varying $\beta$, that is for any crack geometry as $\beta$ increases, the stress intensity factors increase. For $\beta \rightarrow \infty$ the results shown in the table agree with that of a half plane containing a uniformly pressurized crack of length $2 \mathrm{c}$ perpendicular to the boundary. As the distance $d$ increases, the stress intensity factors tend to $\sigma \sqrt{c}$, the homogeneous plane solution.

Figures 2-4 show the variation of the normalized stress intensity factors defined by

$$
k(a)=\frac{k_{1}(a)}{\sigma \sqrt{c}}, k(b)=\frac{k_{1}(b)}{\sigma \sqrt{c}}
$$

with the crack distance $d$ and for various values of the nonhomogeneity parameter $\beta$. For the

${ }^{4}$ The expression (64a) is valid also for the limiting case of $a=0$. In fact (3b) and (4a) implies that $\partial u / \partial x$ is continuous across $x=0$. Since $\partial v / \partial y, \mu$ and $\kappa$ are also continuous, this means that $\sigma_{1 y y}(-0, y)=\sigma_{2 y y}(+0, y), y \geq 0$, implying that the stress singularity will be controlled by that of $\phi(x)$ and the values of $\mu$ and $\kappa$ at $x=0, y=0$. 
specific crack geometry $a=0$, the effect of $\beta$ on the normalized stress intensity factors are shown in Fig. 5. In this figure the absissa is selected in such a way that the results include the full range of $\beta,-\infty<\beta<\infty$. As seen from the figure for $\beta \rightarrow \infty$ the stiffness of medium 1 approaches zero, $k(a) \rightarrow \infty$ and $k(b)$ goes to the edge crack solution, 1.5861 marked on the figure. Similarly, for $\beta \rightarrow \infty$ medium 1 becomes a rigid half space, $k(b)$ goes to the appropriate limit shown in the figure and $k(a)$ tends to zero.

Finally, Fig. 6 shows some examples giving the normalized crack surface displacement for the limiting case of $a=0$ defined by

$$
\mathrm{V}(\mathrm{x})=\frac{\mathrm{v}_{2}(\mathrm{x},+0)}{(1+\kappa) \sigma \mathrm{c} / 4 \mu_{\mathrm{o}}}
$$

The dashed line shown corresponds to $\beta=0$ or the homogeneous medium. Again, as expected the displacement is skewed toward or away from the interface depending on the sign of $\beta$ or the relative stiffnesses of materials 1 and 2 .

Acknowledgement. This study was supported by NSF under the grant MSM-8613611, by NASA-Langley under the grant NAG-1-713 and by SRC contract 88-MP-071.

\section{References}

1. Atkinson, C., 1977, "On Stress Singularities and Interfaces in Linear Elastic Fracture Mechanics", International Journal of Fracture, Vol. 13, pp. 807-820.

2. Comninou, M., 1977, "The Interface Crack", ASME Journal of Applied Mechanics, Vol. 44, pp. 631-636.

3. Cook, T.S. and Erdogan, F., 1972, "Stresses in Bonded Materials with a Crack Perpendicular to the Interface", Int. J. Engng. Sci., Vol. 10, pp. 677-697.

4. Delale, F. and Erdogan, F., 1983, "The Crack Problem for a Nonhomogeneous Plane", ASME Journal of Applied Mechanics, Vol. 50, pp. 609-614.

5. Delale, F. and Erdogan, F., 1988a, "Interface Crack in a Nonhomogeneous Medium", Int. J. Engng. Sci., Vol. 26, pp. 559-568.

6. Delale, F. and Erdogan, F., 1988b, "On the Mechanical Modelling of the Interfacial Region in Bonded Half Planes", ASME Journal of Applied Mechanics, Vol. 55, pp. 317324.

7. England, A.H., 1965, “A Crack Between Dissimilar Media”, ASME Journal of Applied Mechanics, Vol. 32, pp. 400-402.

8. Erdogan, F., 1965, "Stress Distribution in Bonded Dissimilar Materials with Cracks", 
ASME Journal of Applied Mechanics, Vol. 32, pp. 403-410.

9. Erdogan, F. and Biricikoglu, V., 1973, "Two Bonded Half Planes with a Crack Going Through the Interface", Int. J. Engng. Sci., Vol. 11, pp. 745-766.

10. Erdogan, F., and Cook, T.S., 1974, "Antiplane Shear Crack Terminating at and Going Through a Bimaterial Interface", International Journal of Fracture, Vol. 10, pp. 227240.

11. Erdogan, F., 1985, "The Crack Problem for Bonded Nonhomogeneous Materials Under Antiplane Shear Loading", ASME Journal of Applied Mechanics, Vol. 52, pp. 823-828.

12. Muskhelishvili, N.I., 1953a, Some Basic Problems of the Mathematical Theory of Elasticity, P. Noordhoff Ltd., Groningen-Holland.

13. Muskhelishvili, N.I., 1953b, Singular Integral Equations, P. Noordhoff, Ltd., GroningenHolland.

14. Rice, J.R. and Sih, G.C., 1965, "Plane Problems of Cracks in Dissimilar Media", ASME Journal of Applied Mechanics, Vol. 32, pp. 418-423. 
Table 1. Normalized mode I stress intensity factors for a crack of length $2 c$ subjected to constant crack surface pressure $\sigma, \nu=0.3$, the case of plane strain.

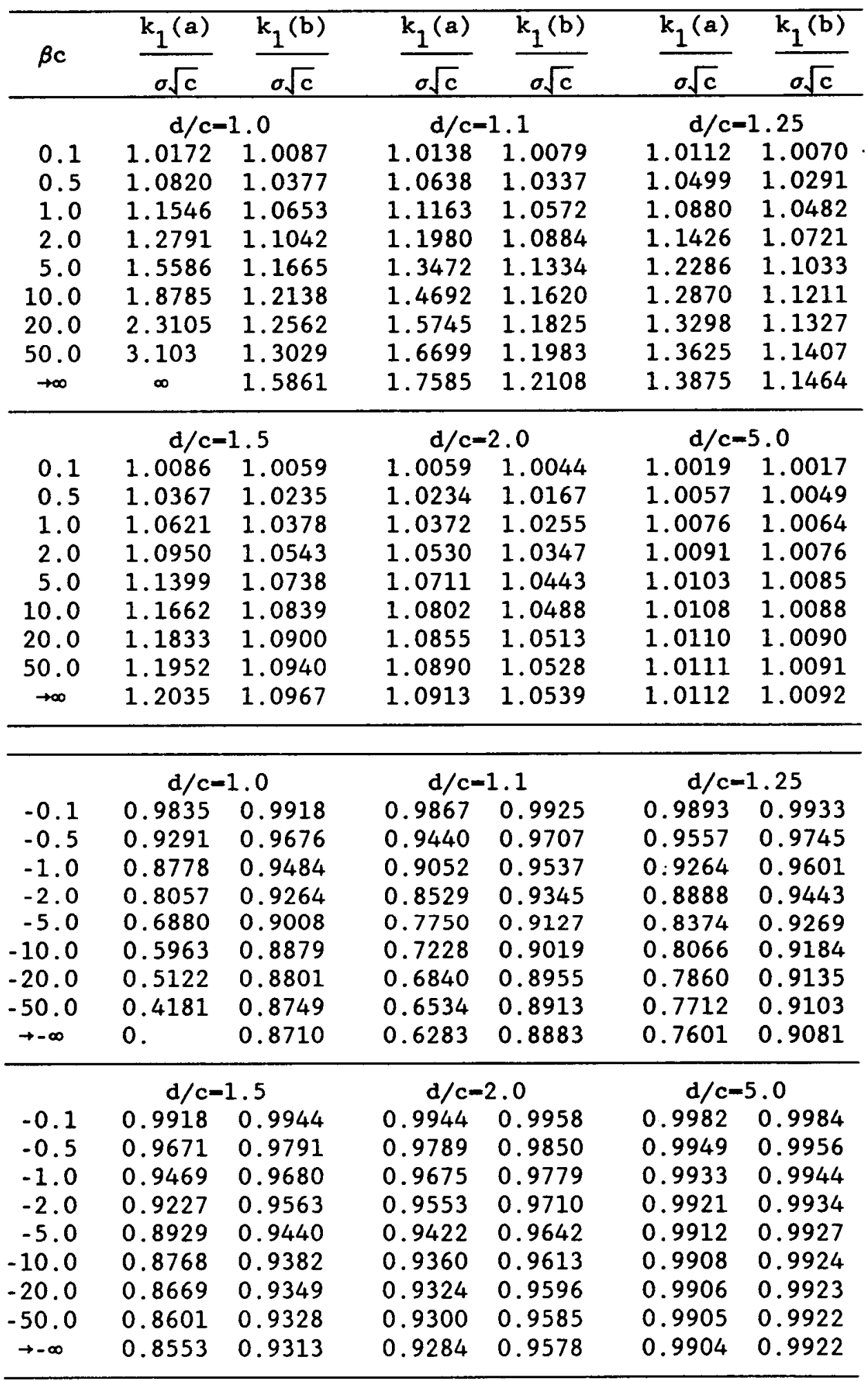




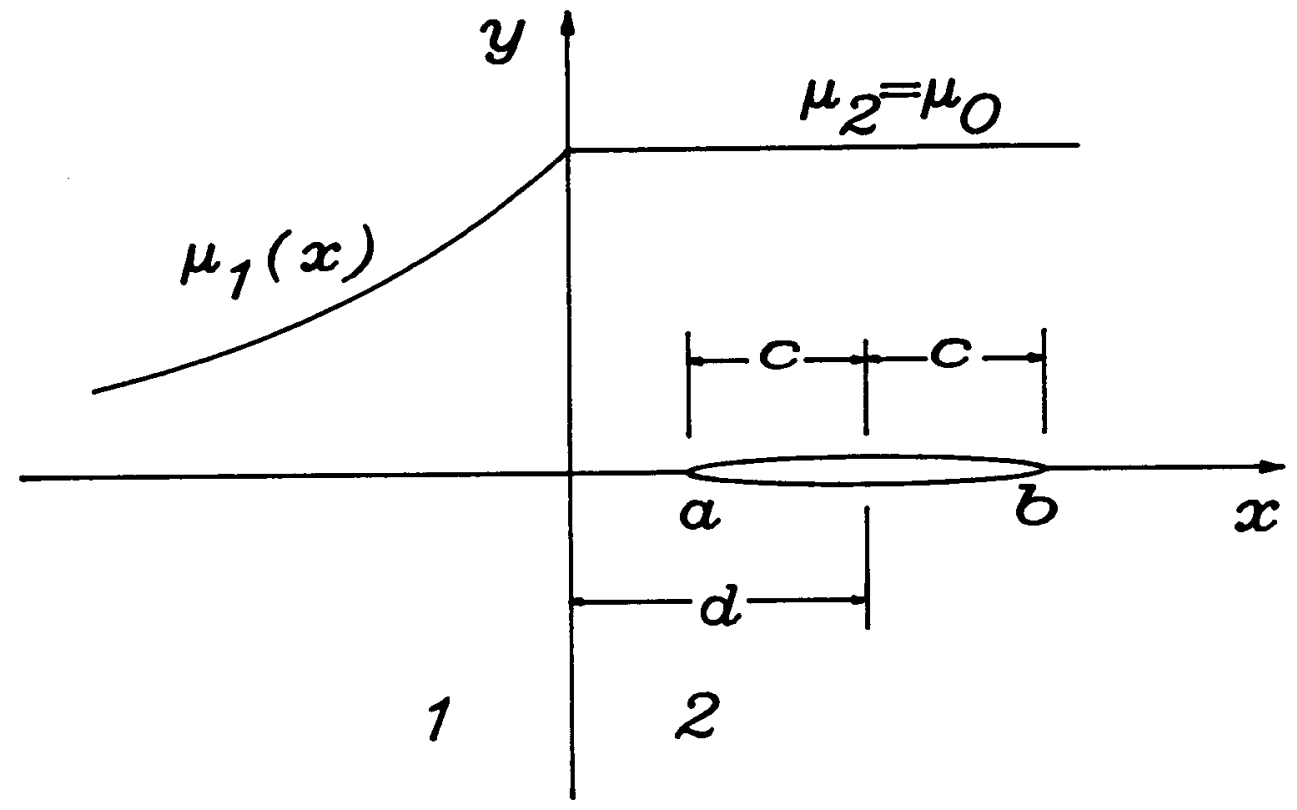

Fig. 1 The crack geometry in bonded materials 

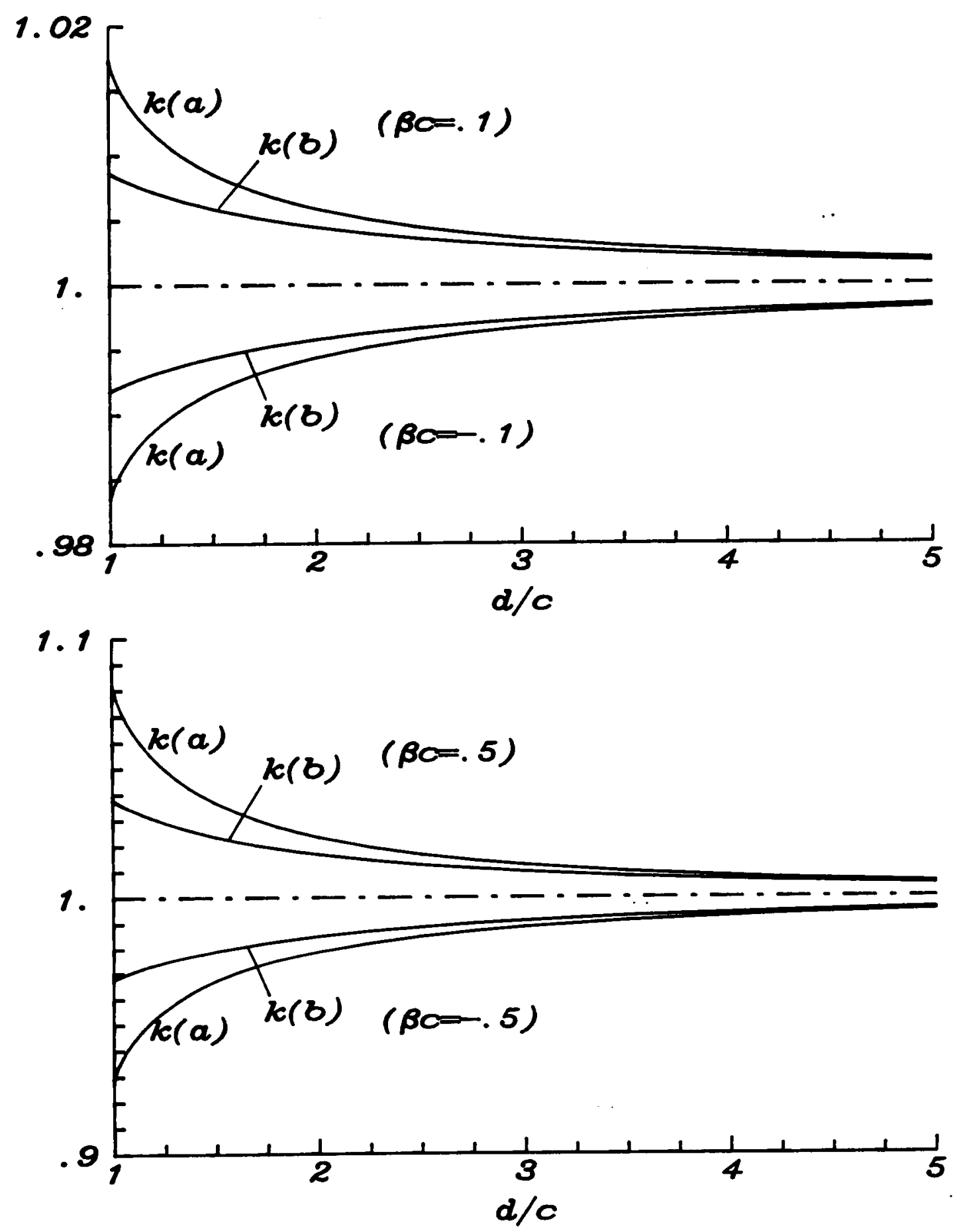

Fig. 2 The variation of the normalized stress intensity factors with the crack distance, $d=(b+a) / 2, c=(b-a) / 2, k(a)=k_{1}(a) / \sigma \sqrt{c}, k(b)=k_{1}(b) / \sigma \sqrt{a}$, the case of plane strain, $\nu=0.3, \beta c=0.1$ and $\beta c=0.5$. 

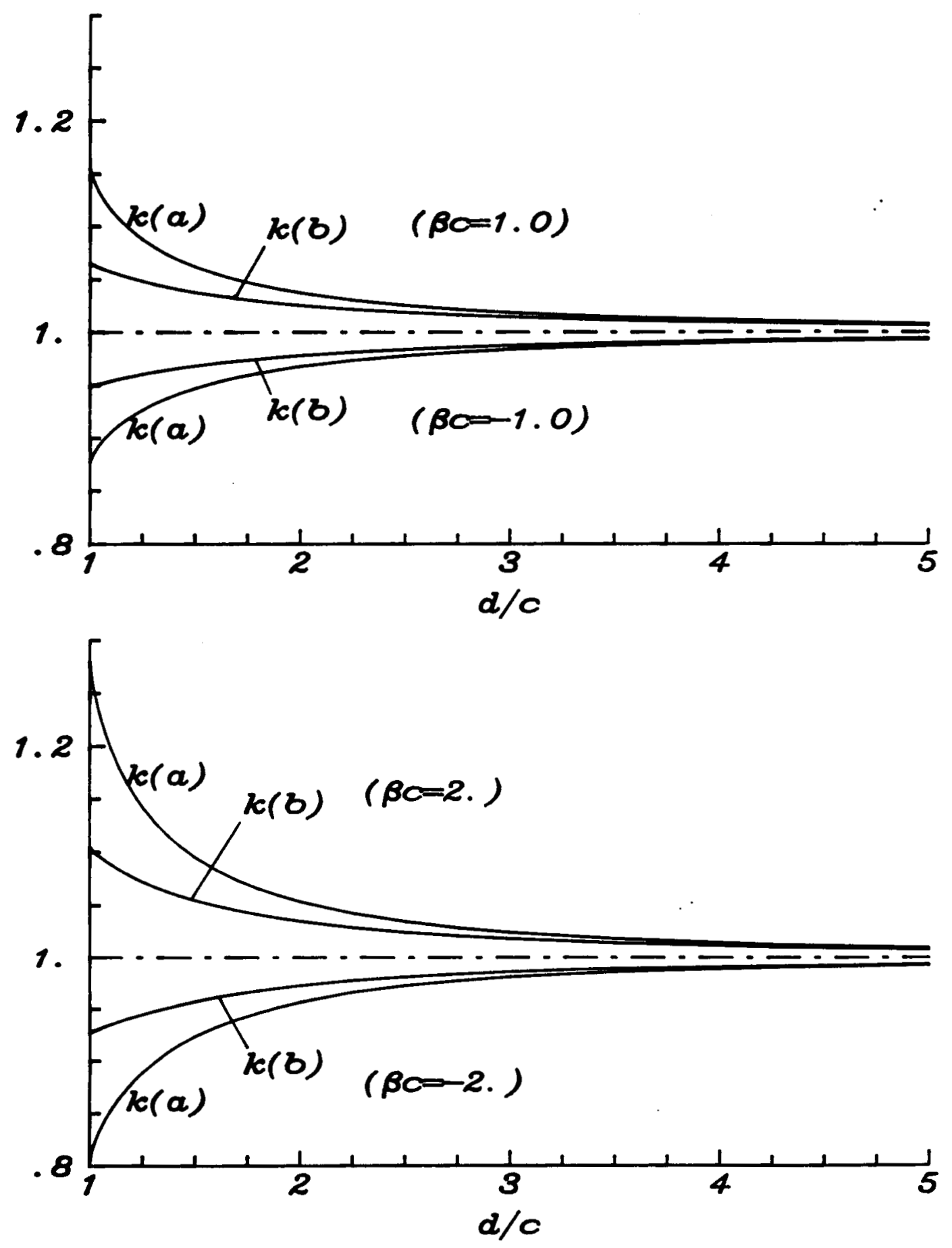

Fig. 3 The variation of the normalized stress intensity factors with the crack distance, $d=(b+a) / 2, c=(b-a) / 2, k(a)=k_{1}(a) / \sigma \sqrt{c}, k(b)=k_{1}(b) / \sigma \sqrt{a}$, the case of plane strain, $\nu=0.3, \beta c=1$ and $\beta c=2$. 

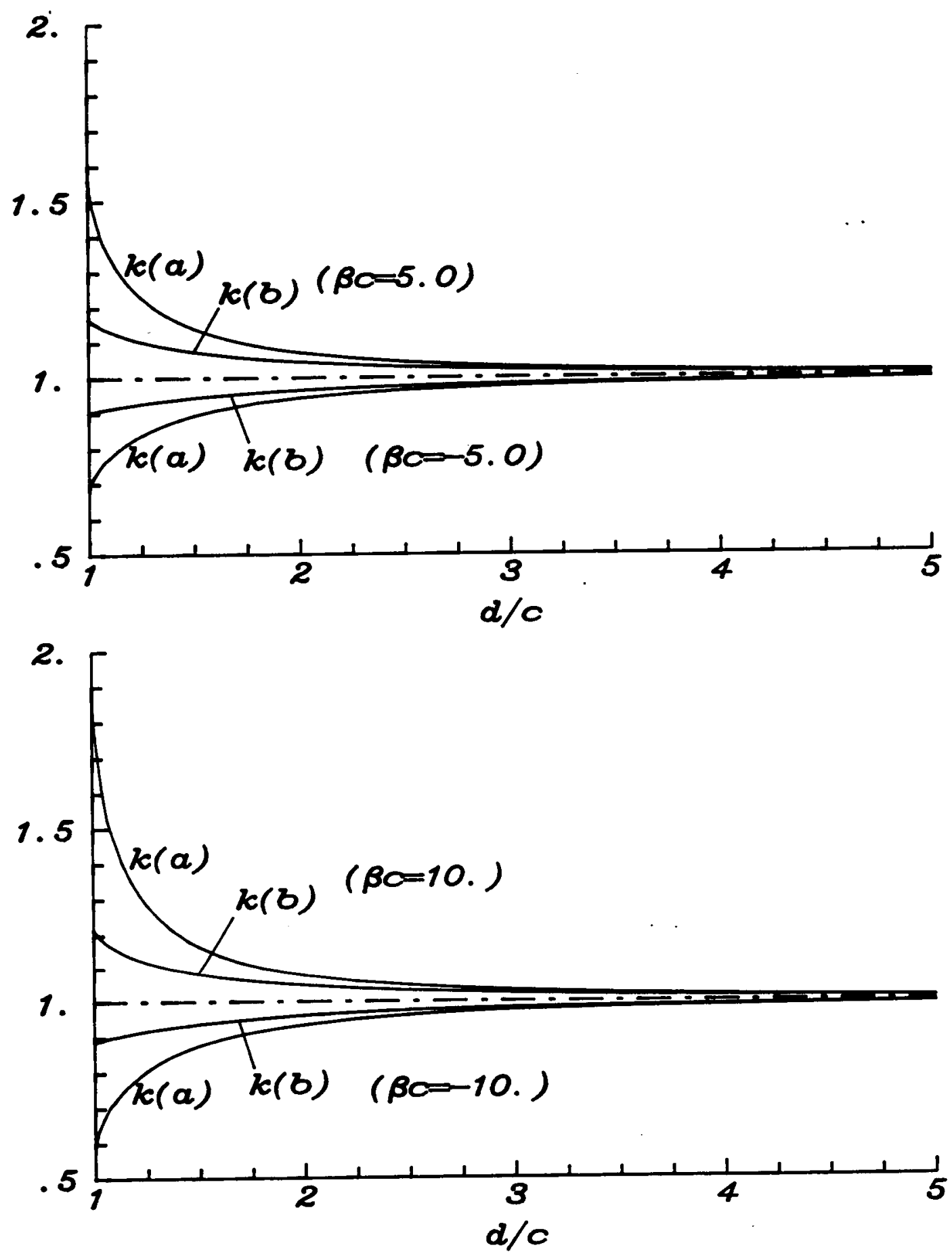

Fig. 4 The variation of the normalized stress intensity factors with the crack distance, $\left.d=(b+a) / 2, c=(b-a) / 2, k(a)=k_{1}\right) / \sigma \sqrt{c}, k(b)=k_{1}(b) / \sigma \sqrt{a}$, the case of plane strain, $\nu=0.3, \beta c=5$ and $\beta c=10$. 


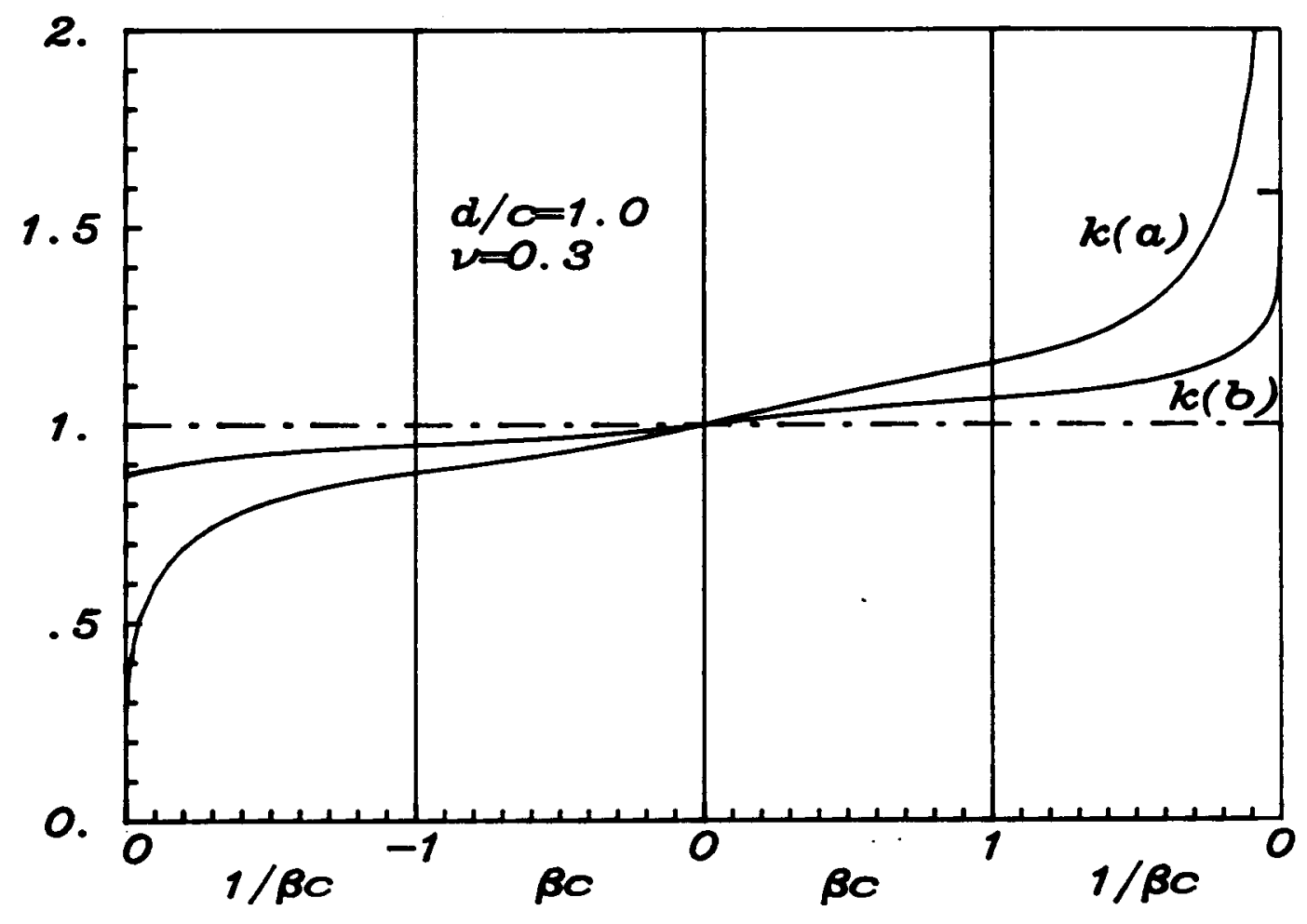

Fig. 5 The variation of the normalized stress intensity factors with the nonhomogeneity constant $\beta$ for the special crack geometry $a=0$. 


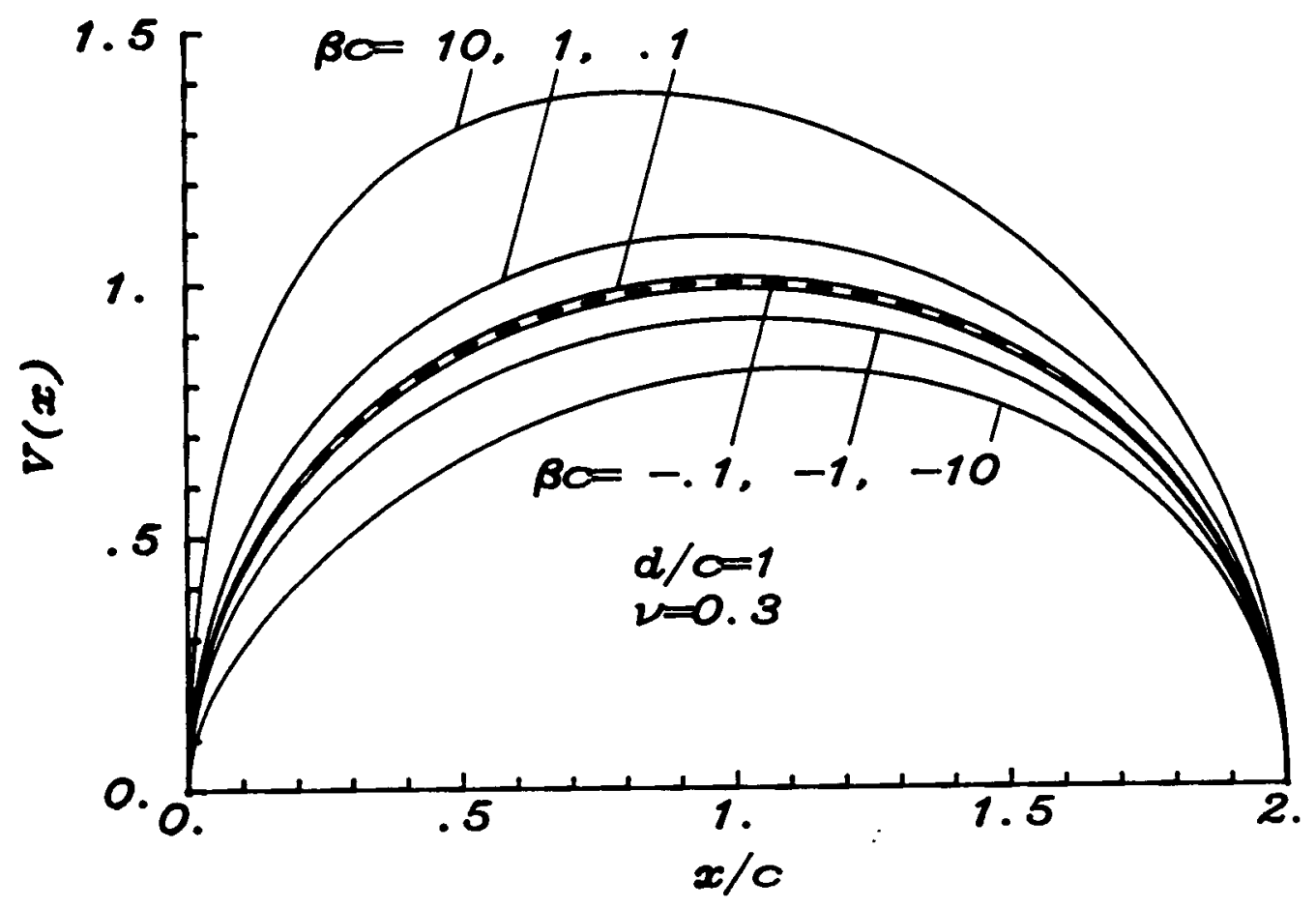

Fig. 6 The normalized crack surface displacement for the special crack geometry $a=0$ and for various values of $\beta, V(x)=v_{2}(x,+0) /\left[(1+\kappa)\left(\sigma c / 4 \mu_{0}\right)\right]$. 


\section{APPENDIX A}

The expressions of the functions $D_{1}, D_{2}, A_{1}$ and $A_{2}$

By substituting from (18), (19b,c), (20), (21b,c) and (23) into (3) and (4), after rather straightforward algebra we obtain

$$
\begin{aligned}
& \mathrm{D}_{1}(\alpha)=\int_{a}^{\mathrm{b}}\left[\mathrm{c}_{1}(\alpha, \mathrm{t})+\mathrm{ic} \mathrm{c}_{2}(\alpha, \mathrm{t})\right] \mathrm{e}^{-\alpha \mathrm{t}} \phi(\mathrm{t}) \mathrm{dt}, \\
& \mathrm{D}_{2}(\alpha)=\int_{\mathrm{a}}^{\mathrm{b}}\left[\mathrm{c}_{1}(\alpha, \mathrm{t})-\mathrm{ic} \mathrm{c}_{2}(\alpha, \mathrm{t})\right] \mathrm{e}^{-\alpha \mathrm{t}} \phi(\mathrm{t}) \mathrm{dt}, \\
& \mathrm{A}_{1}(\alpha)=\int_{\mathrm{a}}^{\mathrm{b}} \mathrm{c}_{3}(\alpha, \mathrm{t}) \mathrm{e}^{-\alpha \mathrm{t}} \phi(\mathrm{t}) \mathrm{dt} \\
& \mathrm{A}_{2}(\alpha)=\int_{a}^{\mathrm{b}} \mathrm{c}_{4}(\alpha, \mathrm{t}) \mathrm{e}^{-\alpha \mathrm{t}} \phi(\mathrm{t}) \mathrm{dt},
\end{aligned}
$$

where

$$
\begin{aligned}
& \mathrm{c}_{\mathrm{k}}(\alpha, \mathrm{t})=\mathrm{p}_{\mathrm{k}}(\alpha)+\mathrm{tq}_{\mathrm{k}}(\alpha),(\mathrm{k}=1,2,3,4) \\
& \mathrm{p}_{1}(\alpha)=\frac{1}{\mathrm{Z}}\left[-\frac{(\kappa-1)^{2}}{(\kappa+1)} \mathrm{B}+\frac{(\kappa-1)(\kappa+3)}{(\kappa+1)} \alpha \mathrm{D}+(\kappa+1)(\mathrm{AD}+\mathrm{BC})\right] \\
& \mathrm{q}_{1}(\alpha)=\frac{2 \alpha}{\mathrm{Z}}\left[-\left(\frac{\kappa-1}{\kappa+1}\right) \mathrm{B}-\left(\frac{\kappa-1}{\kappa+1}\right) \alpha \mathrm{D}-\mathrm{AD}-\mathrm{BC}\right] \\
& \mathrm{p}_{2}(\alpha)=\frac{1}{\mathrm{Z}}\left[-(\kappa-3) \alpha-\frac{(\kappa-1)^{2}}{(\kappa+1)} \mathrm{A}+\frac{(\kappa-1)(\kappa+3)}{(\kappa+1)} \alpha \mathrm{C}+(\kappa+1)(\mathrm{AC}-\mathrm{BD})\right] \\
& \mathrm{q}_{2}(\alpha)=\frac{2 \alpha}{\mathrm{Z}}\left[-\alpha-\left(\frac{\kappa-1}{\kappa+1}\right) \mathrm{A}-\left(\frac{\kappa-1}{\kappa+1}\right) \alpha \mathrm{C}-\mathrm{AC}+\mathrm{BD}\right], \\
& \mathrm{p}_{3}(\alpha)=2 \mathrm{p}_{1}(\alpha)-\frac{1}{2 \alpha}, \\
& \mathrm{q}_{3}(\alpha)=2 \mathrm{q}_{1}(\alpha)+\frac{1}{(\kappa+1)}, \\
& \mathrm{p}_{4}(\alpha)=\frac{2 \alpha}{(\kappa+1) \mathrm{Z}}\left[(\kappa-2) \mathrm{B}-3 \alpha \mathrm{D}-2 \mathrm{AD}-2 \kappa \mathrm{BC}+(\kappa+2) \mathrm{B}\left(\mathrm{C}^{2}+\mathrm{D}^{2}\right)+\frac{\mathrm{D}}{\alpha}\left(\mathrm{A}^{2}+\mathrm{B}^{2}\right)\right] \\
& \mathrm{Z}=2 \alpha\left[-\mathrm{B}+\kappa \alpha \mathrm{D}+2 \kappa \mathrm{AD}+2 \mathrm{BC}-\mathrm{B}\left(\mathrm{C}^{2}+\mathrm{D}^{2}\right)+\frac{\kappa \mathrm{D}}{\alpha}\left(\mathrm{A}^{2}+\mathrm{B}^{2}\right)\right] \\
& \mathrm{q}_{4}(\alpha)=\frac{4 \alpha^{2}}{(\kappa+1) \mathrm{Z}}\left[\mathrm{B}+\alpha \mathrm{D}-\mathrm{B}\left(\mathrm{C}^{2}+\mathrm{D}^{2}\right)-\frac{\mathrm{D}}{\alpha}\left(\mathrm{A}^{2}+\mathrm{B}^{2}\right)\right] \\
& (\mathrm{A} 6-13) \\
&
\end{aligned}
$$

where $A, B, C$ and $D$ are functions of $\alpha$ and are defined by 


$$
A+i B=n_{1}(\alpha), C+i D=m_{1}(\alpha)
$$

and $n_{1}$ and $m_{1}$ are given by (14a) and (12). 


\section{APPENDIX B}

The asymptotic expansions needed to examine the singular behavior of the kernel $K(x, t)$

For $\alpha \rightarrow \infty$ from Appendix A and equations (12), (14), (16) and (28) it can be shown that

$$
\begin{aligned}
& \mathrm{R}(\alpha) \doteq 2 \alpha+\frac{(7-\kappa) \beta^{2}}{4(1+\kappa)} \frac{1}{\alpha}+0\left(\alpha^{-3}\right) \\
& \theta=\frac{1}{2} \sqrt{\frac{3-\kappa}{1+\kappa}}\left[\frac{\beta}{\alpha}-\frac{(15-\kappa) \beta^{3}}{12(1+\kappa)} \frac{1}{\alpha^{3}}+0\left(\alpha^{-5}\right)\right] \\
& \mathrm{A}=\alpha-\frac{\beta}{2}+\frac{\beta^{2}}{2(1+\kappa)} \frac{1}{\alpha}+0\left(\frac{1}{\alpha^{3}}\right) \\
& \mathrm{B}=\frac{1}{2} \sqrt{\frac{3-\kappa}{1+\kappa}}\left[\beta-\frac{\beta^{3}}{2(1+\kappa)} \frac{1}{\alpha^{2}}+0\left(\frac{1}{\alpha^{4}}\right)\right], \\
& \mathrm{C}=-1-\frac{(2-\kappa) \beta}{2} \frac{1}{\alpha}-\frac{\left(\kappa^{3}-2 \kappa^{2}-3 \kappa+2\right) \beta^{2}}{4(1+\kappa)} \frac{1}{\alpha^{2}}+\frac{(1+\kappa)(2-\kappa)(3-\kappa) \beta^{3}}{8} \frac{1}{\alpha^{3}}+0\left(\frac{1}{\alpha^{4}}\right) \\
& \mathrm{D}=\frac{1}{2} \sqrt{\frac{3-\kappa}{1+\kappa}}\left[\kappa \beta \frac{1}{\alpha}+\frac{(1+\kappa)(2-\kappa) \beta^{2}}{2} \frac{1}{\alpha^{2}}+\frac{\left(\kappa^{4}-\kappa^{3}-5 \kappa^{2}-\kappa+4\right) \beta^{3}}{4(1+\kappa)} \frac{1}{\alpha^{3}}+0\left(\frac{1}{\alpha^{4}}\right)\right] \\
& \mathrm{Z}=4 \sqrt{\frac{3-\kappa}{1+\kappa}}(1-\kappa)(1+\kappa)\left[-\beta \alpha \cdot \frac{(1-\kappa)(2+\kappa) \beta^{2}}{2(1+\kappa)}+0\left(\frac{1}{\alpha}\right)\right] \\
& \mathrm{h}_{1}(\alpha) \doteq \frac{\beta}{4 \alpha}+0\left(\frac{1}{\alpha^{2}}\right) \\
& \mathrm{h}_{2}(\alpha) \cong 0\left(\frac{1}{\alpha^{2}}\right) \\
& \mathrm{h}_{3}(\alpha) \tilde{=}-\frac{\beta}{(1+\kappa)}+\frac{(1-\kappa) \beta^{2}}{2(1+\kappa)^{2}} \frac{1}{\alpha}+0\left(\frac{1}{\alpha^{2}}\right),
\end{aligned}
$$




$$
\mathrm{h}_{4}(\alpha) \tilde{=} \frac{\beta}{1+\kappa} \alpha-\frac{(1-\kappa) \beta^{2}}{2(1+\kappa)^{2}}-\frac{\kappa(3-\kappa) \beta^{3}}{4(1+\kappa)^{3}} \frac{1}{\alpha}+0\left(\frac{1}{\alpha^{2}}\right)
$$




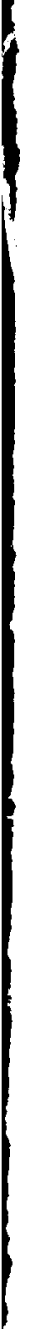

